\title{
miR-133a-3p inhibits the osteogenic differentiation of bone marrow mesenchymal stem cells by regulating ankyrin repeat domain 44
}

\author{
Mao Li ${ }^{1}$, Ya-Jun Shen'2, Shuai Chai ${ }^{3}$, Yu-Long Bai ${ }^{4}$ and Zhong-Hai Li ${ }^{5}$ \\ ${ }^{1}$ Department of Orthopedics, Beijing Tongren Hospital, Capital Medical University, Dongcheng District, Beijing, China \\ ${ }^{2}$ Beijing Institute of Dental Research, Beijing Key Laboratory, Beijing Stomatological Hospital and School of Stomatology, \\ Capital Medical University, Beijing, China \\ ${ }^{3}$ Department of Neurosurgery, Wenxi People Hospital of Shanxi Province, China \\ ${ }^{4}$ Research and Invention Center, Shanghai Yapeng Biological Technology Co. LTD, Shanghai Yapeng Biotechnology Co. LTD, \\ China \\ ${ }^{5}$ Department of Orthopedics, The First Affiliated Hospital of Dalian Medical University, Dalian, China
}

\begin{abstract}
In this study, we aimed to identify the specific microRNAs (miRNAs) that are involved in the osteogenic differentiation of bone marrow mesenchymal stem cells (BMSCs) from ovariectomized (OVX) mice, and to further explore the mechanism by which these miRNAs regulate osteogenic differentiation. Based on the existing studies, the expression of seven miRNAs in BMSCs from OVX mice was evaluated using quantitative reverse transcription polymerase chain reaction (qRT-PCR). The expression of miR-133a-3p and osteogenesis-related genes (runt-related transcription factor 2 (Runx2), Osterix, alkaline phosphatase (ALP), and osteopontin) in BMSCs treated with miR-133a-3p mimics or inhibitors was detected by qRT-PCR or Western blotting. Osteogenesis efficiency was determined using ALP and alizarin red staining. The effector-target relationship between miR-133a-3p and ankyrin repeat domain 44 (ANKRD44) was confirmed by bioinformatics and a dual luciferase assay. Among the seven selected miRNAs, miR-133a-3p expression was significantly increased in BMSCs from OVX mice. Overexpression of miR-133a-3p dramatically inhibited the expression of osteogenesis-related genes in BMSCs and reduced ALP activity and mineralization. However, these processes were markedly ameliorated upon miR-133a-3p inhibition. Moreover, miR-133a-3p appeared to target $A N K R D 44$, and the ANKRD44 expression was negatively regulated by miR-133a3p. Furthermore, ANKRD44 upregulation eliminated the anti-osteogenic differentiation effects of miR-133a-3p in BMSCs. Thus, our results indicated that miR-133a-3p inhibits the osteogenic differentiation of BMSCs by suppressing ANKRD44.
\end{abstract}

Key words: miR-133a-3p - Osteogenic differentiation - BMSCs - ANKRD44

\section{Introduction}

Postmenopausal osteoporosis (PMO) is a systemic and metabolic disease of the bones; it is caused in response to the decreased estrogen levels in older women. PMO is

Correspondence to: Zhong-Hai Li, Department of Orthopedics, The First Affiliated Hospital of Dalian Medical University, No. 5, Longbin Road, Dalian Development Zone, Dalian, Liaoning Province, China E-mail: lizhonghaispine@126.com characterized by reduced bone mass and micro-architectural deterioration of the bone tissue, and increased bone fragility and the risk of fracture (Black and Rosen 2016; Yang et al. 2020). In addition to affecting the normal activity of the individual and increasing the mortality, PMO also imposes a heavy economic burden (Harvey et al. 2010). At present, approximately $30 \%$ of all postmenopausal women in the United States and Europe have osteoporosis (Reginster and Burlet 2006). It is estimated that the standardized prevalence in women in mainland China will increase from $26.10 \%$ in 
2020 to $39.19 \%$ in 2050 (Cui et al. 2019b). The mechanism underlying the development of PMO involves an imbalance between bone resorption and bone formation (Dimitriou et al. 2011).

It has been noted that osteogenesis is a key factor in the process of bone formation (Collins and Stratakis 2016). Bone marrow mesenchymal stem cells (BMSCs) are a class of adult stem cells that are present in the bone marrow stroma and participate in the formation of the bone marrow microenvironment by virtue of their multipotency and active proliferative properties (Pilz et al. 2011). As the progenitor cells of osteoblasts, BMSCs play critical roles in bone formation and differentiation, serving as an excellent source of seed cells in bone tissue engineering (Chen et al. 2016). Functional defects, such as reduced proliferative activity and decreased osteogenic differentiation of BMSCs, are important factors that contribute to the onset of PMO (Fan et al. 2014).

MicroRNAs (miRNAs) are small non-coding RNA molecules (approximately 22-24-nt long). They are involved in many important biological processes and regulate gene expression at the post-transcriptional level by targeting the 3'-untranslated region (3'-UTR) of messenger RNAs (mRNAs). Abnormal miRNA expression can result in the development of a variety of diseases (Czech 2006). Notably, increasing number of studies show that miRNAs play an important role in osteoclast differentiation (Hrdlicka et al. 2019). Recent studies have indicated that miR-130a, miR-185, and miR-141-3p are involved in BMSC osteogenesis. miR-130a promotes the osteoblastic differentiation of BMSCs by negatively regulating Smurf2 expression (Lin et al. 2019). miR-185 depletion promotes osteogenic differentiation and suppresses bone loss in osteoporosis through the biglycan-mediated bone morphogenetic protein/Smad pathway (Cui et al. 2019a). It is also noteworthy that miR141-3p inhibits osteogenic differentiation in both human and murine BMSCs by modulating the expression of stromal cell-derived factor 1 (Periyasamy-Thandavan et al. 2019). These findings clearly demonstrate the critical role of miRNAs in BMSC osteogenesis. However, the role of miRNAs in the osteogenic differentiation of BMSCs requires further clarification.

The reliability of ovariectomized (OVX) animals as postmenopausal osteoporosis models has previously been demonstrated (Frost and Jee 1992). Some studies have investigated the changes in the miRNA expression profile in the bones of mice with OVX-induced osteoporosis using microarray analysis (An et al. 2014; Hao et al. 2016). In the present study, we selected seven differentially expressed miRNAs in OVX mice based on the results of microarray analyses in previous studies, with an aim to identify the specific miRNAs involved in the osteogenic differentiation of BMSCs. Further, we also investigated the mechanism by which miRNAs regulate osteogenic differentiation.

\section{Materials and Methods}

\section{OVX mouse model}

Animal studies were reviewed and approved by the Animal Care and Use Committee of Beijing Tongren Hospital, Capital Medical University, and were performed according to the guidelines adhered by the hospital. Thirty 8 -week-old C57BL/6 mice were randomly divided into either the OVX group $(n=10)$ or the sham group $(n=10)$. The mice were anesthetized with a $50 \mathrm{mg} / \mathrm{kg}$ dose of sodium pentobarbital via intraperitoneal injection. In OVX group mice, both ovaries were removed, whereas in the sham group, only a portion of the adipose tissue around the ovaries was removed.

\section{BMSC isolation, culture, and transfection}

C57BL/6 mice between the ages of 6 and 8 weeks were euthanized by cervical dislocation. BMSCs were isolated from the femurs of mice and cultured in conditional medium ( $\alpha$-minimal essential medium ( $\alpha$-MEM) containing 10\% fetal calf serum (FCS)) as previously described (Maridas et al. 2018). Passage three cells were used for the subsequent experiments.

miR-133a-3p mimics (5'-UUUGGUCCCCUUCAACCAGCUG-3'), negative control (NC) mimics (5'-UUUUCCGAACGUUCACGUTT-3'), miR-133a-3p inhibitors (5'-CAGCUGGUUGAAGGGGACCAAA-3'), and NC inhibitors (5'-CAGUACUUUUGUGUAGUACAA-3') were purchase from GenePharm (Shanghai, China). ANKRD44 (NM_001081433.3) was expressed using the pcDNA3.1 vector (Addgene, Watertown, MA, USA), and cells transfected with the empty pcDNA3.1 vector were used as the NC. BMSCs cultured to $80 \%$ confluence in a 6-well plate $\left(1 \times 10^{6}\right.$ cells/well $)$ were transfected with mimics, inhibitors, pcDNA3.1-ANKRD44, or the empty pcDNA3.1 vector using Lipofectamine 2000 (Invitrogen, Carlsbad, CA, USA) according to the manufacturer's instructions. After transfection, the cells were cultured in osteogenic medium (a-MEM containing $10 \% \mathrm{FCS}, 10^{-8} \mathrm{M}$ dexamethasone, $10 \mathrm{mM} \beta$-glycerol phosphate, and $50 \mathrm{mg} / \mathrm{ml} \mathrm{L}$-ascorbic acid) for 7 days. Then, the subsequent experiments were performed.

\section{Alkaline phosphatase (ALP) staining and quantitative analysis}

ALP staining was performed using an ALP Staining Kit (Beyotime $^{\circledast}$ Institute of Biotechnology, Shanghai, China) according to the manufacturer's protocol. Briefly, cells were washed twice with phosphate-buffered saline (PBS) and incubated in ALP staining solution (Sigma-Aldrich, St. Louis, $\mathrm{MO}, \mathrm{USA}$ ) at $37^{\circ} \mathrm{C}$ for $20 \mathrm{~min}$ with gentle shaking in the dark. 
ALP activity was determined by quantifying the amount of p-nitrophenol - the yellow-colored end product of p-nitrophenyl phosphate hydrolysis - using an Alkaline Phosphatase Assay Kit (Beyotime ${ }^{\circledast}$ Institute of Biotechnology). The quantity of ALP in the cell lysates was measured at $405 \mathrm{~nm}$ using a microplate reader (ELX800; BioTek, Winooski, VT, USA).

\section{Alizarin red staining (ARS) and quantitative analysis}

Cells were washed with PBS and fixed in 4\% paraformaldehyde (Sigma-Aldrich) for $10 \mathrm{~min}$ at room temperature. After removing the fixative, the cells were rinsed with double-distilled $\mathrm{H}_{2} \mathrm{O}$ and stained with $40 \mathrm{mM}$ alizarin red (Sigma-Aldrich, St. Louis, MO, USA) at pH 4.2 for $30 \mathrm{~min}$ with gentle agitation at room temperature.

The cells were then rinsed with double-distilled $\mathrm{H}_{2} \mathrm{O}$ to remove the excess dye and subsequently imaged. In order to elute the bound dye for quantification, the stained cells were incubated with $800 \mu \mathrm{l}$ of acetic acid for $30 \mathrm{~min}$ at room temperature, and absorbance was measured using a microplate reader (ELX800; BioTek) at $570 \mathrm{~nm}$.

\section{Quantitative reverse transcription polymerase chain reaction} (qRT-PCR)

For mRNA analysis, total RNA was isolated from the cells using TRIzol (Invitrogen) according to the manufacturer's protocol. qRT-PCR was performed using a one-step RT-PCR kit (QIAGEN, Germany) according to the manufacturer's instructions. Relative expression was calculated for each gene using the $2^{-\Delta \Delta \mathrm{Ct}}$ method following normalization against $\beta$-actin (NM_007393) expression.

The primers used in this study are as follows: Osterix (forward: 5'-TCAAGTGGCATAGATGTGGAAGAA-3', reverse: 5'- TGGCTCTGCAGGATTTTCATG-3'), ALP (forward: 5'-TCTTCTCATTCCTGCTTGTGG-3', reverse: 5'-GGTCTGGGCCATAGAACTGA-3'), runt-related transcription factor 2 (Runx2; forward: 5'-ATTGGCACCATCTTTACTGTTACC-3', reverse: 5'-CTCCTTAGAATCTGTTTGCTCTCATA-3'), osteopontin (OPN; forward: 5'-CTGACAAAGCCTTCATGTCCAA-3', reverse: 5'-CCGCACGACAACCGCACCAT-3'), GAPDH (forward: 5'-TGGCACCCAGCACAATGAA-3', reverse: 5'-CTAAGTCATAGTCCGCCTAGAAGCA-3'), and $\beta$-actin (forward: 5'-GCACCACACCTTCTACAATG-3', reverse: 5'-TGCTTGCTGATCCACATCTG-3').

For miRNA analysis, total RNA was isolated from the cells according to protocol for RNAiso for Small RNA (Takara Bio, Beijing, China). RNA (1 $\mu \mathrm{g})$ was reverse transcribed using the Bulge-Loop ${ }^{\mathrm{TM}}$ miRNA RT Primer (RiboBio, Guangzhou, China), and qPCR was performed on a Roche Light Cycler System using the Bulge-Loop miRNA
qRT-PCR starter kit (RiboBio). Relative expression was calculated for each miRNA using the $2^{-\Delta \Delta \mathrm{Ct}}$ method following normalization against small nuclear RNA U6 expression.

The qPCR primer sequences used in this study are as follows: miR-15b-5p (forward: 5'-TAGCAGCACATCATGGTTTACA-3'), miR-205-5p (forward: 5'-TCCTTCATTCCACCGGAGTCTG-3'), miR-27a-3p (forward: 5'-TTCACAGTGGCTAAGTTCCGC-3'), miR-483-5p (forward: 5'-ACACTCCAGCTGGGAAGACGGGAGGAA-3'), miR133a-3p (forward: 5'-ACACTCCAGCTGGGTTTGTCCCCTTCAAC-3'), miR-17-5p (forward: 5'-GCCGCCAAAGTGCTTACA-3'), miR-342-3p (forward: 5'-TCTCACACAGAAATCGCACCCGT-3'), universal miRNA reverse primer proprietary sequence (B661601; Sangon Biotech, Shanghai, China), and U6 (forward: 5'-CTCGCTTCGGCAGCACA-3', reverse: 5'-AACGCTTCACGAATTTGCGT-3').

The PCR protocol consisted of two steps, 1) initial denaturation for $30 \mathrm{~s}$ at $95^{\circ} \mathrm{C}$ and 2) denaturation for $5 \mathrm{~s}$ at $95^{\circ} \mathrm{C}$, annealing and extension for $31 \mathrm{~s}$ at $60^{\circ} \mathrm{C}$, and fluorescence signal acquisition. The reactions were performed for a total of 40 cycles and ended with the generation of a melt curve, which consisted of $15 \mathrm{~s}$ at $95^{\circ} \mathrm{C}, 1 \mathrm{~min}$ at $60^{\circ} \mathrm{C}, 15 \mathrm{~s}$ at $95^{\circ} \mathrm{C}$, and $15 \mathrm{~s}$ at $60^{\circ} \mathrm{C}$. PCR product specificity was confirmed by melt curve analysis.

\section{Dual-luciferase reporter assay}

wt and mt ANKRD44 3'-UTR sequences were obtained by PCR amplification using a template and primers, and were subsequently cloned into the SpeI and HindIII sites of the pMIR-REPORT Luciferase miRNA Expression Reporter Vector (Applied Biosystems, Carlsbad, CA, USA). The resulting construct was co-transfected ( $5 \mathrm{ng}$ ) into $293 \mathrm{~T}$ cells along with $20 \mathrm{nM}$ control or miR-133a-3p mimics using Lipofectamine 2000 (Invitrogen) according to the manufacturer's instructions. After $24 \mathrm{~h}$ of transfection, luciferase activity in the cells was determined using the Luciferase Assay System (Promega, Madison, WI, USA).

\section{Western blotting}

After transfection for 7 days, BMSCs were collected and analyzed via Western blotting. The cells were lysed in radioimmunoprecipitation assay buffer containing proteinase inhibitors. Protein samples were separated on $10 \%$ gels by sodium dodecyl sulfate polyacrylamide gel electrophoresis. The proteins were electroblotted onto polyvinylidene difluoride membranes $(0.45 \mathrm{~mm}$; Millipore, Billerica, MA, USA). Next, the membranes were blocked using 5\% nonfat dry milk prepared in Tris-buffered saline containing Tween 20 for $1 \mathrm{~h}$. The membranes were then probed overnight with the following primary antibodies: anti-Runx2 
antibody (1:1000; \#12556; Cell Signaling Technology, Boston, MA, USA), anti-OPN antibody (1:1000; ab8448; Abcam, Cambridge, UK), anti-Osterix antibody (1:1000; ab209484; Abcam), anti-ALP antibody (1:500; ab67228; Abcam), and anti-GAPDH antibody (1:2500; ab9485; Abcam) at $4^{\circ} \mathrm{C}$. This was followed by incubation with an HRP-conjugated secondary antibody (1:2000; ab97051; Abcam) at room temperature for $1 \mathrm{~h}$. Protein bands were visualized using an enhanced chemiluminescence system, and the levels of the experimental proteins were normalized to those of actin. The levels of phosphorylated proteins were normalized to those of the corresponding total proteins.

\section{Target prediction}

The target genes of miR-133a-3p were predicted using two bioinformatic algorithms, i.e., ECROI (http://starbase.sysu. edu.cn/agoClipRNA.php? source $=$ mRNA) and miRDB (http://www.mirdb.org/mining.html).

\section{Statistical analysis}

All statistical analyses were performed using SPSS Statistics 11.0 software (IBM, Armonk, NY, USA). All data are expressed as means \pm standard deviations. The Student's $t$-test or a one-way analysis of variance followed by Turkey's post hoc test was used to analyze the data. $p<0.05$ was considered to be significant.

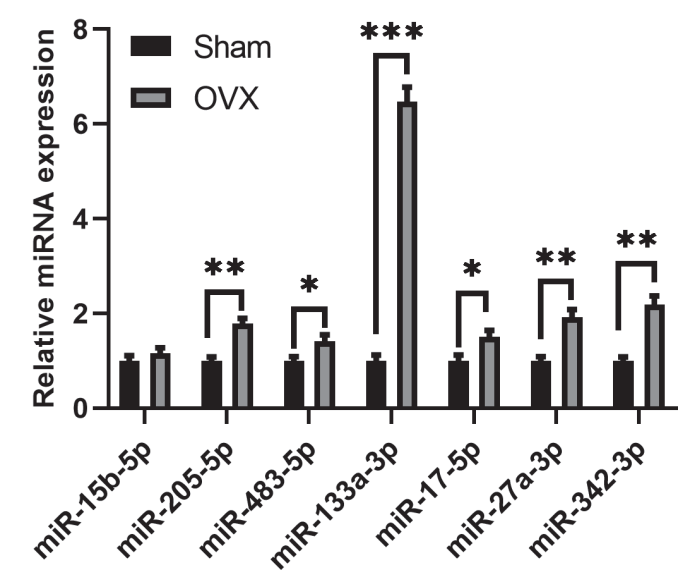

Figure 1. Expression of miRNAs in BMSCs from OVX or sham mice. The qRT-PCR results of the expression levels of seven predicted miRNAs in BMSCs isolated from OVX or sham mice $(n=5)$. Data are presented as means \pm SD based on three independent experiments. ${ }^{*} p<0.05,{ }^{* *} p<0.01,{ }^{* *} p<0.001 v s$. Sham group. miRNA, microRNA; BMSCs, bone marrow mesenchymal stem cells; OVX, ovariectomized group; Sham, sham operation group.

\section{Results}

Expression of seven predicted miRNAs in BMSCs from OVX mice

To identify the specific differentially expressed miRNAs in BMSCs from OVX mice, we selected seven miRNAs that were differentially expressed in the bone tissues of OVX mice based on the findings of previous studies. qRT-PCR revealed that among these seven miRNAs, miR-205-5p, miR-483-5p, miR-133a-3p, miR-17-5p, miR-27a-3p, and miR-342-3p were upregulated in BMSCs from OVX mice compared to BMSCs from the sham group (Fig. 1). Of note, the expression of miR-133a-3p was significantly higher in BMSCs from OVX mice.

Suppression of osteoblast differentiation via forced miR133a-3p expression

To investigate the effect of miR-133a-3p overexpression on osteogenic differentiation, we used miR-133a-3p mimics, and cells treated with the scrambled sequence were used as a parallel control. qRT-PCR confirmed that the expression of miR-10b was dramatically upregulated in the mimics group compared to that in the NC group (Fig. 2A). Transfected BMSCs were induced to differentiate into the osteoblast lineage using osteogenic induction media. qRTPCR revealed that miR-133a-3p mimic-transfected BMSCs exhibited lower levels of Runx2, Osterix, ALP, and OPN than the control cells (Fig. 2B). Western blotting revealed that the protein levels of these markers were also decreased in miR-133a-3p mimic-transfected BMSCs (Fig. 2C). The ALP staining assay showed that the expression of ALP - an early marker of osteoblasts - was decreased in response to miR-133a-3p overexpression (Fig. 2D). ALP activity was also decreased upon miR-133a-3p overexpression (Fig. 2E). Alizarin red staining and quantification indicated that matrix mineralization was reduced in the miR-133a-3p mimics group (Fig. 2F and G). These data demonstrated that miR-133a-3p overexpression inhibits the osteogenic differentiation of BMSCs in vitro.

\section{Enhancement of osteoblast differentiation via miR-133a-3p downregulation}

In order to further explore the effects of miR-133a-3p on osteoblast differentiation, we suppressed the expression of miR-133a-3p in BMSCs using inhibitors (Fig. 3A). The results showed that miR-133a-3p downregulation markedly increased the mRNA expression of two osteoblast differentiation-related transcription factors, i.e., Runx 2 and Osterix, as well as those of known osteoblast differentiation markers ALP and OPN (Fig. 3B). Similar changes were also 

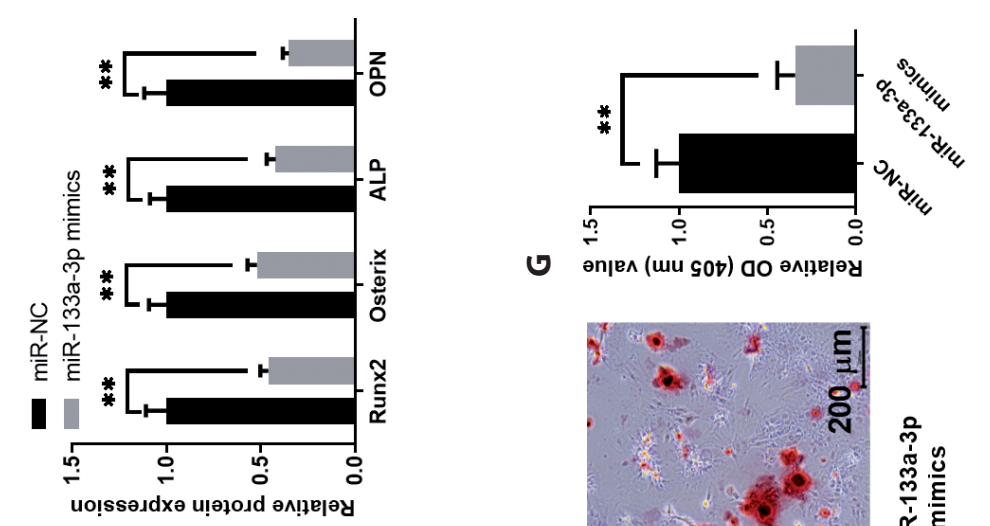

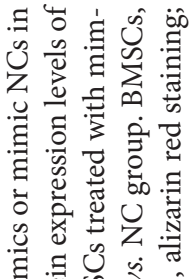

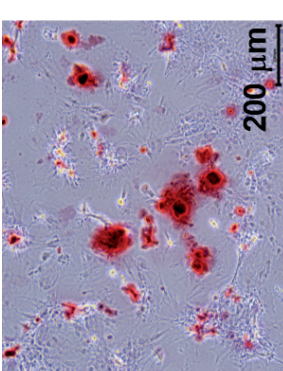

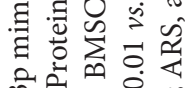

कि

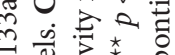

m. $\quad \frac{m}{1}$

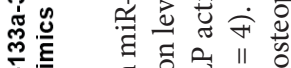

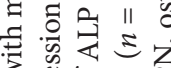

ङ
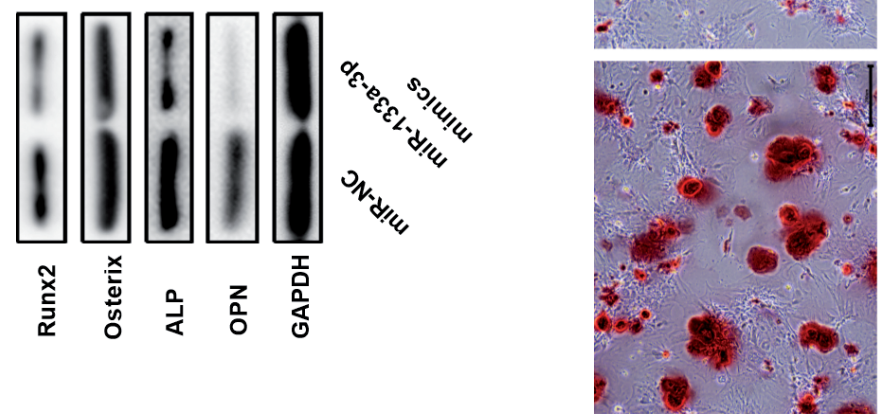

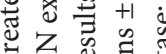

芯过芯焉

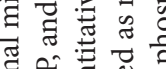

펴ำ

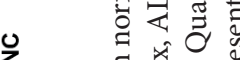

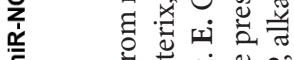

ह क ज

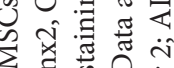

$\sum_{0}$ क 0

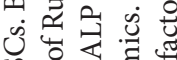

น

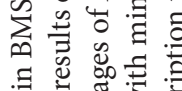

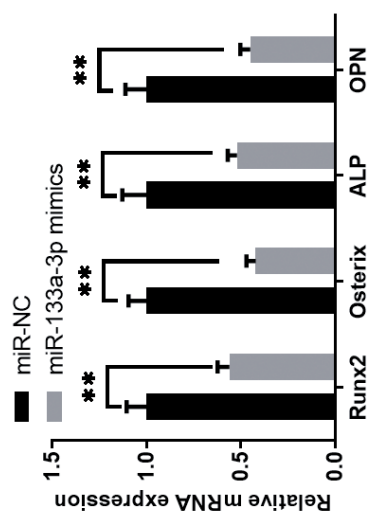

ш

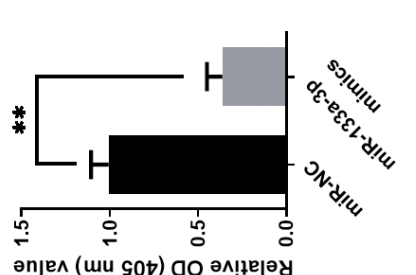

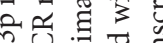

के

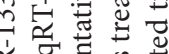

घี

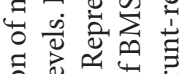

10

仓ั

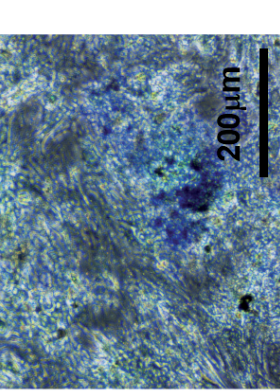

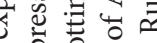

엉응 흥

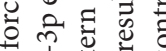

o․ :

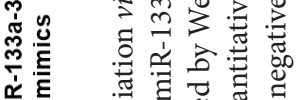

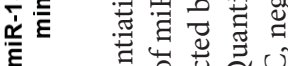

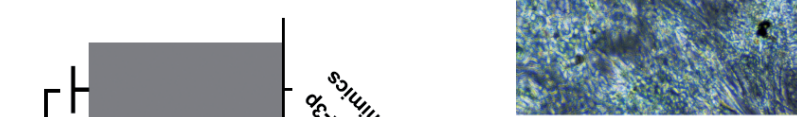

¿

矛节苛

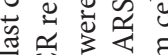

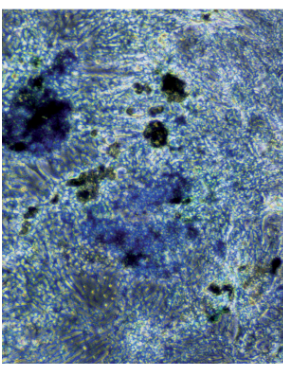

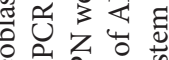

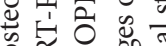

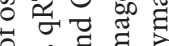

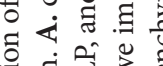

U

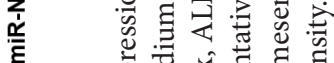

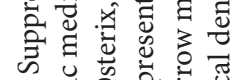

क

ป

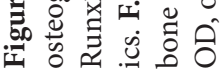



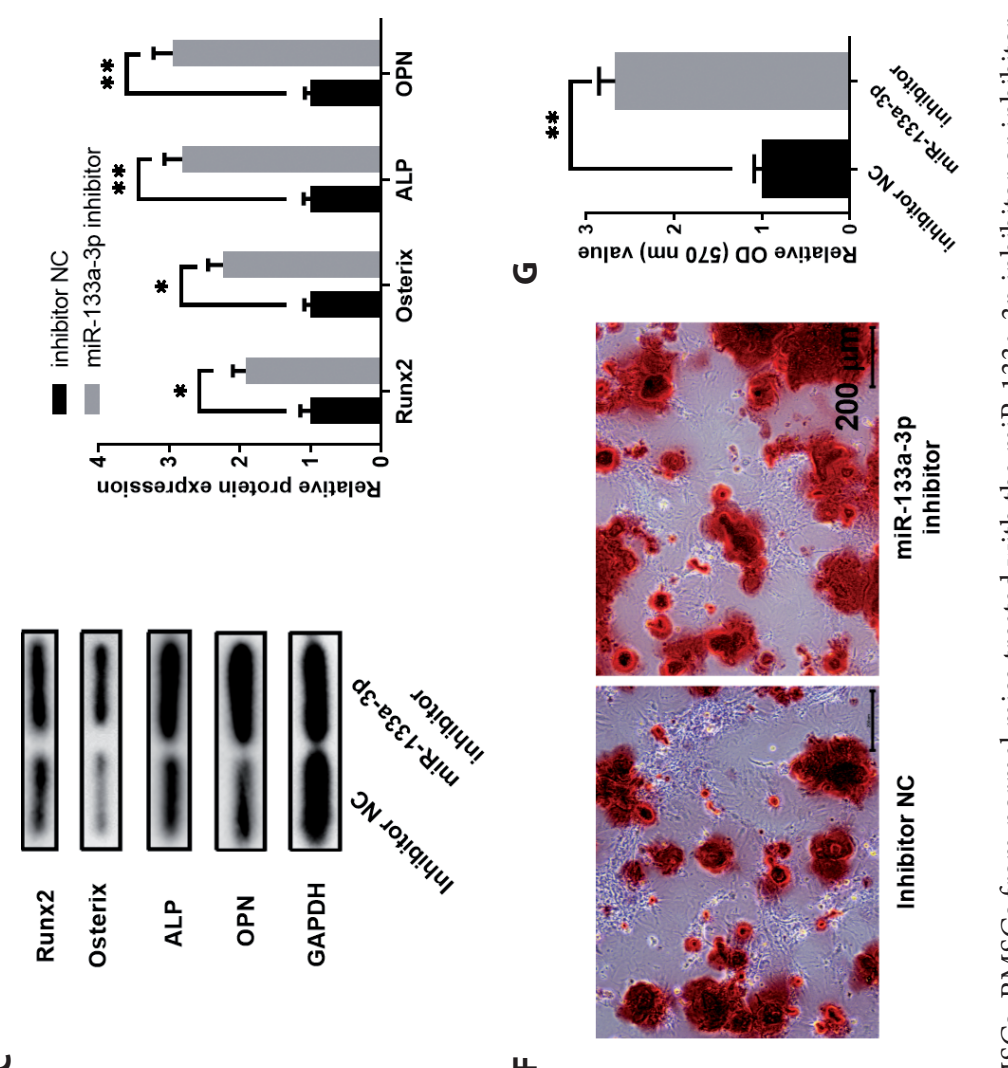

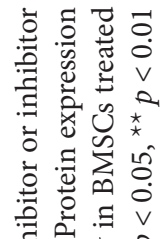
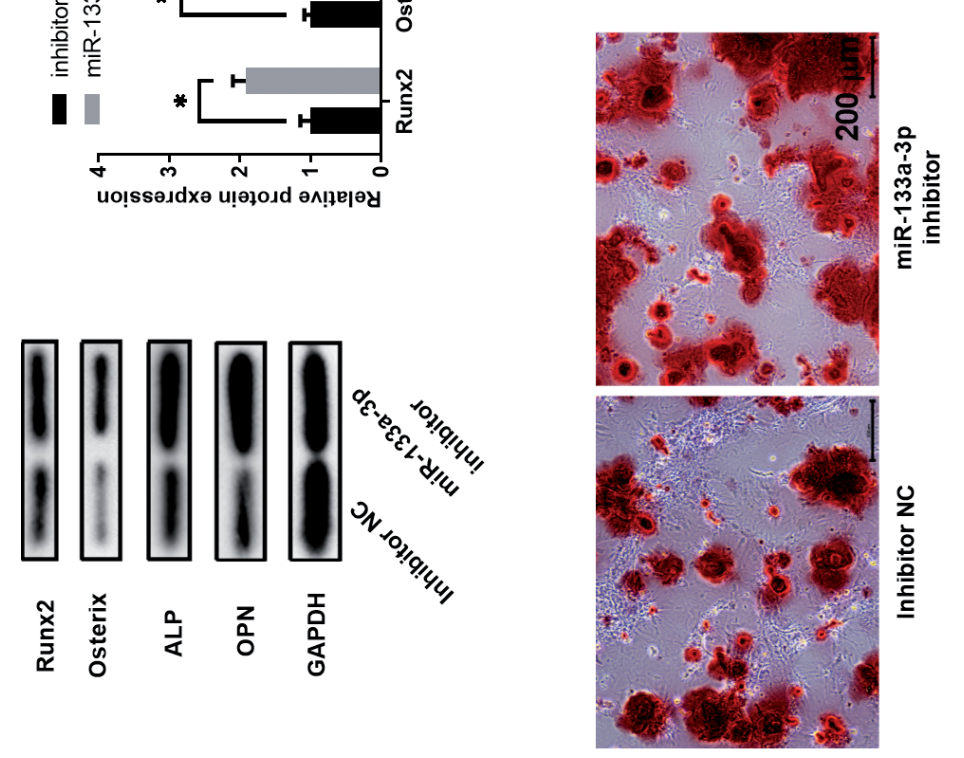

U

ले एं

ले ?

五安会

है 㐘

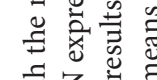

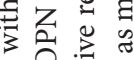

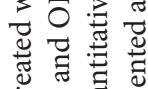

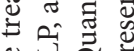

岁菅

छิ xิ

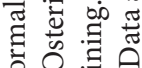

$\exists$ 跔

茟害完

出出出吉

$\sum_{0}^{\infty} \stackrel{0}{0}$

में

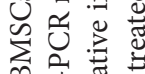

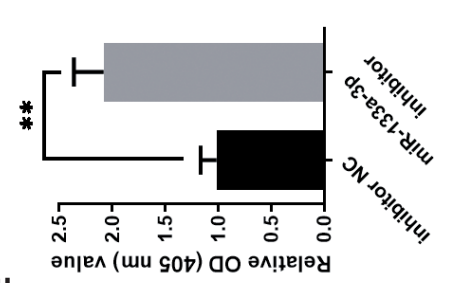

$\Xi$ 崫苛

ธี

ज्ञ

कृ

हี

원응 븍

के

ले के क्षे

वें

元

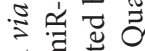

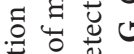

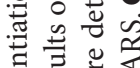

¿ 仓

車出 $Z$ 品

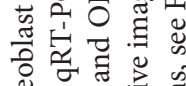

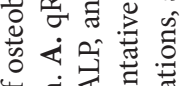

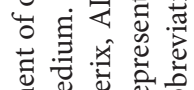

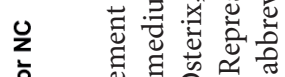

웜 पु

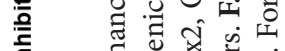

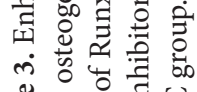

四

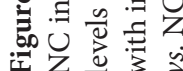


observed at the protein level for these molecules through Western blotting (Fig. 3C). ALP staining in the miR-133a-3p inhibitor-transfected cells was significantly increased compared to that in the miR-133a-3p inhibitor NC-transfected cells (Fig. 3D). ALP activity was increased upon miR-133a-3p inhibition (Fig. 3E). ARS staining and quantification were also markedly enhanced in the miR-133a-3p-transfected cells (Fig. 3F-G). These results further confirmed that miR- 133a-3p downregulation can enhance the differentiation of BMSCs into osteoblasts.

\section{Direct targeting of the ANKRD44 3'-UTR by miR-133a-3p}

To identify the targets that mediate the osteoblast differentiation inhibitory effect of miR-133a-3p, we predicted the potential targets in silico using various databases. Upon
A

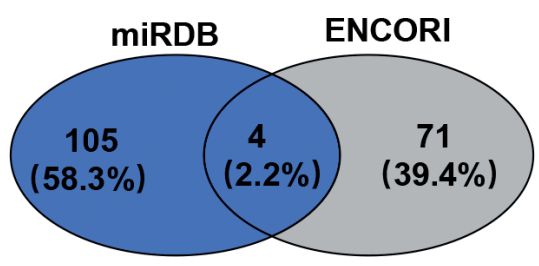

C

WT ANKRD44 3'UTR 5' ACCAGCUCUCCAUGAGGACCAAA MUT ANKRD44 3'UTR 5' ACCAGCUCUCCAUGAACUAGAAA miR-133a-3p 3' GUCGACCAACUUCCCCUGGUUU
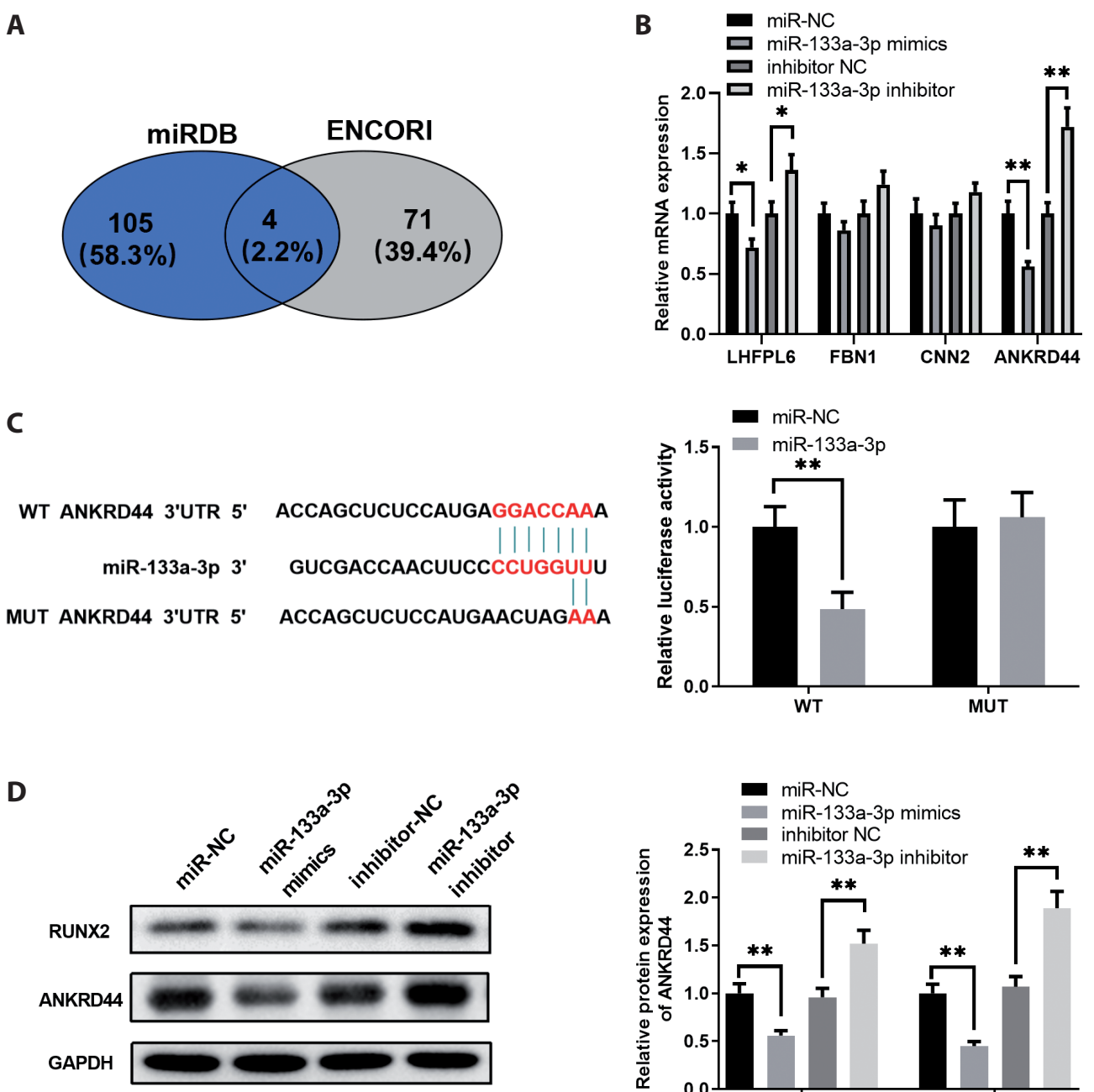

E

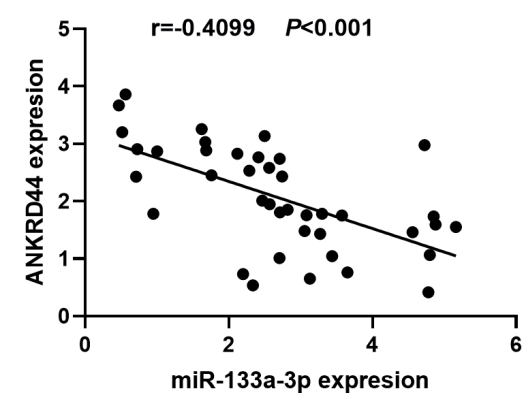

Figure 4. Direct targeting and expression regulation of the 3 ' UTR of ANKRD44 by miR-133a-3p. A. The binding seed sequence was predicted by miRDB and ENCORI. B. Effects of miR-133a-3p mimics and inhibitors on mRNA expression levels. C. A luciferase reporter assay was performed to confirm the matching of miR-133a$3 p$ and the 3' UTR of ANKRD44. D. Western blotting analyzed the effect of miR-133a-3p on ANKRD44 expression. E. Negative linear relationship between the miR-133a-3p and ANKRD44 expression. Data are presented as means $\pm \mathrm{SD}(n=4){ }^{*} p<0.05$, ${ }^{* *} p<0.01 v s$. NC group. UTR, untranslated region; ANKRD44, ankyrin repeat domain 44; mRNA, messenger RNA; NC, negative control. 
considering the complementarity of the miRNA seed sequence, the miRDB as well as the ENCORI database revealed that $A N K R D 44$ contains a binding site for the miR-133a-3p seed sequence in its 3'-UTR (Fig. 4A), and that miR-133a-3p regulated ANKRD44 expression as detected by PCR (Fig. 4B). To further confirm the interaction between the miR-133a-3p seed sequence and the 3'-UTR of its potential target genes, a luciferase reporter assay was performed (Fig. 4C). The results showed that miR-133a$3 \mathrm{p}$ directly binds to the 3'-UTR of the ANKRD44 mRNA without targeting the 3 '-UTR of ANKRD44 mRNA contain- ing a mutant seed region or the 3'-UTRs of other genes as mentioned previously. We then analyzed the regulatory effect of miR-133a-3p on the expression of its potential target ANKRD44. Consistent with the results of the luciferase reporter assay, western blotting revealed that the miR-133a-3p mimic decreased ANKRD44 expression (Fig. $4 \mathrm{D})$. In addition, there was a negative linear relationship between the miR-133a-3p and ANKRD44 expression (Fig. $4 \mathrm{E})$. These data suggested that miR-133a-3p can bind to the 3'-UTR of ANKRD44 mRNA and regulate its expression at the post-transcriptional level in BMSCs.
A

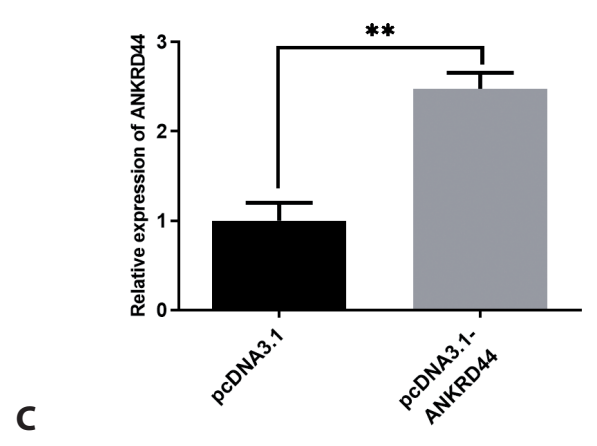

B

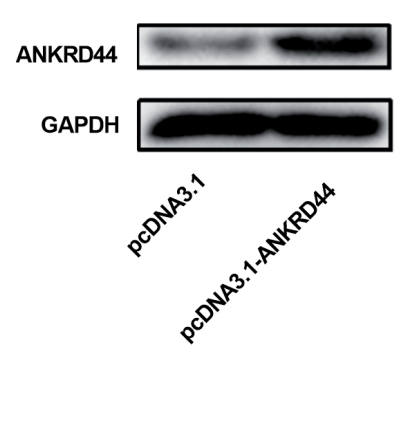

D
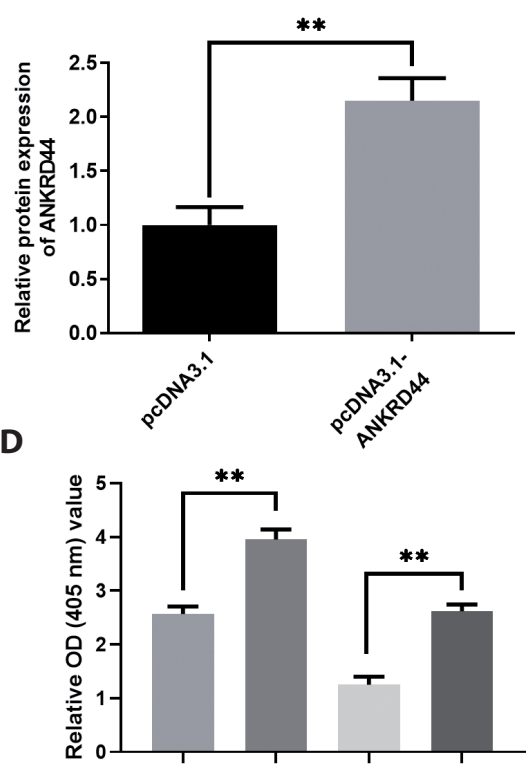

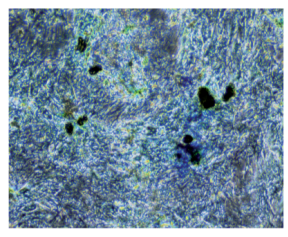

miR-133a-3p mimics +pcDNA3.1

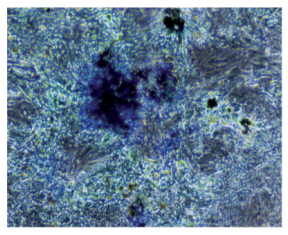

miR-NC+pcDNA3.1

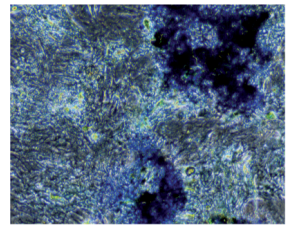

miR-NC+ pcDNA3.1-ANKRD44
miR-133a-3p mimics+ pCDNA3.1-ANKRD44
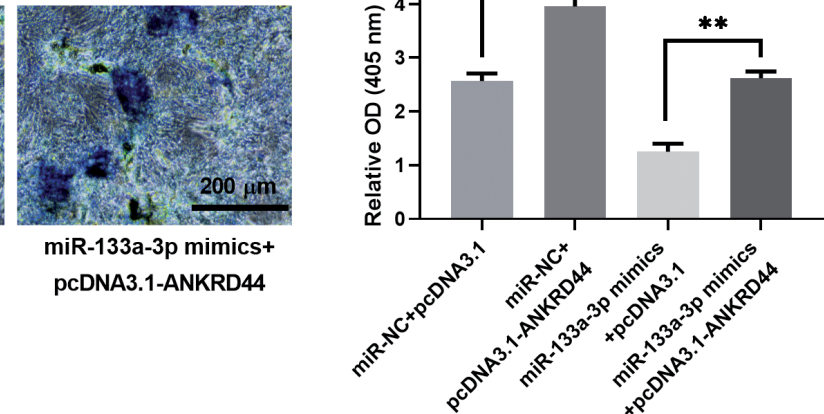

$\mathbf{F}$

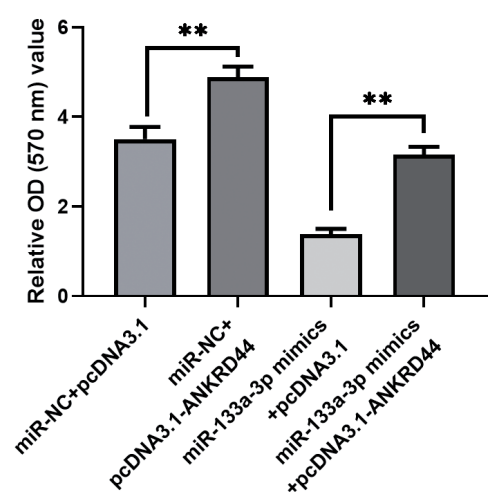

Figure 5. Overexpression of ANKRD44 counteracts the inhibition of miR-133a-3p on BMSC osteogenic differentiation in vitro. BMSCs from normal mice were cultured in OM (miR-NC+pcDNA3.1, miR-133a-3p mimics+pcDNA3.1, and miR-133a-3p mimics+pcDNA3.1ANKRD44). A. qRT-PCR analysis of ANKRD44 expression. B. Western blot analysis of ANKRD44 protein expression. C. Representative images of ALP staining. D. Quantitative results of ALP activity. E. Representative images of ARS. F. Quantitative results of ARS. Data are presented as means $\pm \mathrm{SD}(n=4) ;{ }^{* *} p<0.01$. ANKRD44, ankyrin repeat domain 44. For more abbreviations, see Fig. 2. 
Overexpression of ANKRD44 counteracts miR-133a-3p-mediated inhibition of BMSC osteogenic differentiation in vitro

To further confirm the essential role of ANKRD44 in miR-133a-3p-mediated inhibition of osteogenic differentiation, we expressed the entire ANKRD44 coding sequence using an expression vector in BMSCs. ANKRD44 mRNA expression in BMSCs transfected with pcDNA3.1ANKRD44 or the pcDNA3.1 empty vector was analyzed via qRT-PCR. Indeed, the expression of ANKRD44 was considerably elevated in the pcDNA3.1-ANKRD44 group at the mRNA and protein levels (Fig. 5A and B). Notably, miR-133a-3p-mediated inhibition of osteogenic differentiation was significantly rescued by ANKRD44 overexpression as evidenced by the results of the ALP and ARS assays (Fig. $5 \mathrm{C}-\mathrm{F}$ ). These findings further demonstrated that miR-133a$3 p$ inhibits BMSC osteogenic differentiation mechanically via ANKRD44 suppression.

\section{Discussion}

PMO is a systemic and metabolic bone disorder that is caused as a result of estrogen deficiency-induced disruption of the balance between osteoclast bone resorption and osteoblast bone formation. It is well known that BMSCs are the source of osteoblasts in the bone tissue. The reduced osteogenic differentiation of BMSCs was identified in patients with PMO as well as in a PMO mouse model (Lindtner et al. 2014; Shuai et al. 2015), which implies that the abnormal osteogenic differentiation of BMSCs may contribute to the occurrence and development of PMO. However, the regulatory mechanism underlying the development of PMO remains unclear.

An increasing number of studies have indicated that miRNAs play a vital role in the osteogenic differentiation of BMSCs (Wang et al. 2019). Based on microarray and enrichment analyses from previous studies, we selected seven miRNAs that were upregulated in OVX mice and found that miR-133a-3p was highly expressed in BMSCs from OVX mice. miR-133a-3p has been identified to be downregulated - and to display tumor suppressive functions - in many types of human malignancies, including gastric cancer (Zhang et al. 2018), colorectal cancer (Yu et al. 2019), esophageal squamous cell carcinoma (Yin et al. 2019), and breast cancer (Shi et al. 2019). In addition, previous studies have reported that miR-133a-3p expression was significantly increased in OVX mice (Hao et al. 2016; Wang et al. 2017), which was also confirmed in our study. Our qRT-PCR results showed that miR-133a-3p expression was significantly higher in BMSCs in OVX mice than that in the BMSCs of the sham group, which suggested that miR-133a-3p may play an important role in the osteogenic differentiation of BMSCs. A recent meta-analysis also suggested that miR-133a-3p may serve as a potential non-invasive biomarker and therapeutic target in PMO (Pala and Denkceken 2019).

It has been demonstrated that ALP and OPN are bonespecific matrix proteins that are related to bone formation (Meng et al. 2016). Runx2 and its downstream signaling mediator Osterix are critical regulators of osteoblast differentiation that enhance the transcription of osteoblast-related genes, including ALP and OPN (Lian and Stein 2003). To further investigate the role of miR-133a-3p in osteogenic differentiation, the mimics and inhibitors of miR-133a-3p were transfected into BMSCs, and the mRNA and protein expression of Runx2, Osterix, ALP, and OPN was detected via qRT-PCR and Western blotting. According to the results, miR-133a-3p acts a negative regulator of osteogenesis as the miR-133a-3p knockdown enhanced the levels of $A L P$, Runx2, Col-1, and OPN - as well as the proportions of mineralization and ALP activity - indicating the osteoblast differentiation potential of human BMSCs, whereas ectopic expression of miR-133a-3p reduced these levels. Meanwhile, increased proportions of mineralization and ALP activity were also found.

However, the mechanism by which miR-133a-3p inhibits the differentiation of BMSCs into osteoblasts still remains unclear. In this study, we screened several candidate downstream target genes of miR-133a-3p and found that $A N$ KRD44 possessed the necessary binding site. miR-133a-3p inhibits ANKRD44 protein levels by directly binding to the 3'-UTR of ANKRD44. After miR-133a-3p inhibition, the protein levels of $A N K R D 44$ increased. Moreover, we conducted an additional investigation regarding whether the suppressed osteoblast differentiation of BMSCs induced by miR-133a$3 \mathrm{p}$ can be restored by ANKRD44 overexpression. The ALP and ARS staining results showed that ectopic miR-133a-5p expression-induced reduced proportions of mineralization and amplified ALP activity could be partially reversed by forced ANKRD44 expression. All of these findings suggested that $\mathrm{miR}-133 \mathrm{a}-3 \mathrm{p}$ controls the osteoblast differentiation of BMSCs by inhibiting ANKRD44.

The ANKRD44 gene encodes for an ankyrin repeat domain that, together with the ANKRD28 and ANKRD52 proteins, consists of a putative regulatory ankyrin repeat subunit of the protein phosphatase 6 complex, which is an essential eukaryotic Ser/Thr phosphatase (Stefansson et al. 2008). However, the underlying mechanism by which ANKRD44 regulates the osteogenic differentiation of BMSCs requires further exploration.

In summary, our results indicated that miR-133a-3p is highly expressed in BMSCs from OVX mice. This increased expression of miR-133a-3p downregulated ANKRD44 and thus inhibited the osteogenic differentiation of BMSCs in OVX mice. This study demonstrated that miR-133a-3p is 
involved in the pathogenesis of PMO, which provides new insights into the potential therapeutic targets for PMO progression.

Ethical approval. All applicable international, national, and/or institutional guidelines for the care and use of animals were followed. This study was approved by the Ethics Committee of Beijing Tongren Hospital, Capital Medical University (Beijing, China).

Conflicts of interest. All authors declare that they have no competing interests.

Acknowledgements. This work was supported by the LiaoNing Revitalization Talents Program (XLYC1807131), the Natural Science Foundation of Liaoning Province (20170540294), and the Basic Scientific Research Projects of the Universities in Liaoning Province (LQ2017022).

\section{References}

An JH, Ohn JH, Song JA, Yang J-Y, Park H, Choi HJ, Kim SW, Kim SY, Park W-Y, Shin CS (2014): Changes of microRNA profile and microRNA-mRNA regulatory network in bones of ovariectomized mice. J. Bone Miner. Res. 29, 644-656 https://doi.org/10.1002/jbmr.2060

Black DM, Rosen CJ (2016): Clinical practice. Postmenopausal osteoporosis. N. Engl. J. Med. 374, 254-262 https://doi.org/10.1056/NEJMcp1513724

Chen Q, Shou P, Zheng C, Jiang M, Cao G, Yang Q, Cao J, Xie N, Velletri T, Zhang X, et al. (2016): Fate decision of mesenchymal stem cells: adipocytes or osteoblasts? Cell Death Differ. 23, 1128-1139 https://doi.org/10.1038/cdd.2015.168

Collins MT, Stratakis CA (2016): Bone formation, growth, and repair. Horm. Metab. Res. 48, 687-688 https://doi.org/10.1055/s-0042-119907

Cui Q, Xing J, Yu M, Wang Y, Xu J, Gu Y, Nan X, Ma W, Liu H, Zhao H (2019a): Mmu-miR-185 depletion promotes osteogenic differentiation and suppresses bone loss in osteoporosis through the Bgn-mediated BMP/Smad pathway. Cell Death Dis. 10, 172 https://doi.org/10.1038/s41419-019-1428-1

Cui Z, Meng X, Feng H, Zhuang S, Liu Z, Zhu T, Ye K, Xing Y, Sun C, Zhou F, Tian Y (2019b): Estimation and projection about the standardized prevalence of osteoporosis in mainland China. Arch. Osteoporos. 15, 2 https://doi.org/10.1007/s11657-019-0670-6

Czech MP (2006): MicroRNAs as therapeutic targets. N. Engl. J. Med. 354, 1194-1195 https://doi.org/10.1056/NEJMcibr060065

Dimitriou R, Jones E, Mcgonagle D, Glannoudis PV (2011): Bone regeneration: current concepts and future directions. BMC Med. 9, 66 https://doi.org/10.1186/1741-7015-9-66

Fan JZ, Yang L, Meng GL, Lin YS, Wei BY, Fan J, Hu HM, Liu YW, Chen S, Zhang JK, et al. (2014): Estrogen improves the proliferation and differentiation of hBMSCs derived from postmenopausal osteoporosis through notch signaling pathway. Mol. Cell Biochem. 392, 85-93

https://doi.org/10.1007/s11010-014-2021-7

Frost, HM, Jee WS (1992): On the rat model of human osteopenias and osteoporoses. Bone Miner. 18, 227-236

https://doi.org/10.1016/0169-6009(92)90809-R

Hao L, Li J, Tian Y, Wu J (2016): Changes in the microRNA profile of the mandible of ovariectomized mice. Cell. Physiol. Biochem. 38, $1267-1287$ https://doi.org/10.1159/000443074

Harvey N, Dennison E, Cooper C (2010): Osteoporosis: impact on health and economics. Nat. Rev. Rheumatol. 6, 99-105 https://doi.org/10.1038/nrrheum.2009.260

Hrdlicka HC, Lee SK, Delany AM (2019): MicroRNAs are critical regulators of osteoclast differentiation. Curr. Mol. Biol. Rep. $5,65-74$ https://doi.org/10.1007/s40610-019-0116-3

Lian, JB, Stein GS (2003): Runx2/Cbfa1: a multifunctional regulator of bone formation. Curr. Pharm. Des. 9, 2677-2685 https://doi.org/10.2174/1381612033453659

Lin Z, He H, Wang M, Liang J (2019). MicroRNA-130a controls bone marrow mesenchymal stem cell differentiation towards the osteoblastic and adipogenic fate. Cell Proliferat. 52, e12688 https://doi.org/10.1111/cpr.12688

Lindtner RA, Tiaden AN, Genelin K, Ebner HL, Manzl C, Klawitter M, Sitte I, Von-Rechenberg B, Blauth M, Richards PJ (2014): Osteoanabolic effect of alendronate and zoledronate on bone marrow stromal cells (BMSCs) isolated from aged female osteoporotic patients and its implications for their mode of action in the treatment of age-related bone loss. Osteoporos Int. 25, 1151-1161 https://doi.org/10.1007/s00198-013-2494-3

Maridas DE, Rendina-Ruedy E, Le PT, Rosen CJ (2018): Isolation, culture, and differentiation of bone marrow stromal cells and osteoclast progenitors from mice. J. Vis. Exp. 131, e56750 https://doi.org/10.3791/56750

Meng J, Ma X, Wang N, Jia M, Bi L, Wang Y, Li M, Zhang H, Xue X, Hou Z, et al. (2016): Activation of GLP-1 receptor promotes bone marrow stromal cell osteogenic differentiation through beta-catenin. Stem Cell Reports 6, 579-591 https://doi.org/10.1016/j.stemcr.2016.02.002

Pala E, Denkceken T (2019): Differentially expressed circulating miRNAs in postmenopausal osteoporosis: a meta-analysis. Biosci. Rep. 39, BSR20190667 https://doi.org/10.1042/BSR20190667

Periyasamy-Thananvan S, Burke J, Mendhe B, Kondrikova G, Kolhe R, Hunter M, Isales CM, Hamrick M W, Hill WD, Fulzele S (2019): MicroRNA-141-3p negatively modulates SDF-1 expression in age-dependent pathophysiology of human and murine bone marrow stromal cells. J. Gerontol. A Biol. Sci. Med. Sci. 74, 1368-1374 https://doi.org/10.1093/gerona/gly186

Pilz GA, Ulrich C, Ruh M, Abele H, Schafer R, Kluba T, Buhring HJ, Rolauffs B, Aicher WK (2011): Human term placenta-derived mesenchymal stromal cells are less prone to osteogenic differentiation than bone marrow-derived mesenchymal stromal cells. Stem Cells Dev. 20, 635-646 
https://doi.org/10.1089/scd.2010.0308

Reginster JY, Burlet N (2006): Osteoporosis: a still increasing prevalence. Bone 38, S4-9

https://doi.org/10.1016/j.bone.2005.11.024

Shi W, Tang T, Li X, Deng S, Li R, Wang Y, Wang Y, Xia T, Zhang Y, Zen K, et al. (2019): Methylation-mediated silencing of miR$133 a-3 p$ promotes breast cancer cell migration and stemness via miR-133a-3p/MAML1/DNMT3A positive feedback loop. J. Exp. Clin. Cancer Res. 38, 429 https://doi.org/10.1186/s13046-019-1400-Z

Shuai B, Shen L, Zhu R, Zhou P (2015): Effect of Qing'e formula on the in vitro differentiation of bone marrow-derived mesenchymal stem cells from proximal femurs of postmenopausal osteoporotic mice. BMC Complement. Altern. Med. 15, 250 https://doi.org/10.1186/s12906-015-0777-2

Stefansson B, Ohama T, Daugherty AE, Brautigan DL (2008): Protein phosphatase 6 regulatory subunits composed of ankyrin repeat domains. Biochemistry 47, 1442-1451 https://doi.org/10.1021/bi7022877

Wang J, Liu S, Li J, Zhao S, Yi Z (2019): Roles for miRNAs in osteogenic differentiation of bone marrow mesenchymal stem cells. Stem Cell. Res. Ther. 10, 197 https://doi.org/10.1186/s13287-019-1309-7

Wang Q, Li Y, Zhang Y, Ma L, Lin L, Meng J, Jiang L, Wang L, Zhou P, Zhang Y (2017): LncRNA MEG3 inhibited osteogenic differentiation of bone marrow mesenchymal stem cells from postmenopausal osteoporosis by targeting miR-133a-3p. Biomed. Pharmacother. 89, 1178-1186 https://doi.org/10.1016/j.biopha.2017.02.090

Yang T-L, Shen H, Liu A, Dong S-S, Zhang L, Deng F-Y, Zhao Q, Deng H-W (2020): A road map for understanding molecular and genetic determinants of osteoporosis. Nat. Rev. Endocrinol. 16, 91-103 https://doi.org/10.1038/s41574-019-0282-7

Yin Y, Du L, Li X, Zhang X, Gao Y (2019): miR-133a-3p suppresses cell proliferation, migration, and invasion and promotes apoptosis in esophageal squamous cell carcinoma. J. Cell Physiol. 234, 12757-12770 https://doi.org/10.1002/jcp.27896

Yu X, Wang D, Wang X, Sun S, Zhang Y, Wang S, Miao R, Xu X, Qu X (2019): CXCL12/CXCR4 promotes inflammation-driven colorectal cancer progression through activation of RhoA signaling by sponging miR-133a-3p. J. Exp. Clin. Cancer Res. 38, 32 https://doi.org/10.1186/s13046-018-1014-X

Zhang X, Li Z, Xuan Z, Xu P, Wang W, Chen Z, Wang S, Sun G, Xu $\mathrm{J}, \mathrm{Xu} Z$ (2018): Novel role of miR-133a-3p in repressing gastric cancer growth and metastasis via blocking autophagy-mediated glutaminolysis. J. Exp. Clin. Cancer Res. 37, 320 https://doi.org/10.1186/s13046-018-0993-y

Received: January 21, 2020

Final version accepted: September 25, 2020 